\title{
Do Perceptual Instructions Lead to Enhanced Performance Relative to Behavioral Instructions?
}

DOI:

10.1080/00222895.2017.1341382

\section{Document Version}

Accepted author manuscript

Link to publication record in Manchester Research Explorer

\section{Citation for published version (APA):}

Brown-Ojeda, C., \& Mansell, W. (2018). Do Perceptual Instructions Lead to Enhanced Performance Relative to Behavioral Instructions? Journal of Motor Behavior, 50(3), 312-320.

https://doi.org/10.1080/00222895.2017.1341382

\section{Published in:}

Journal of Motor Behavior

\section{Citing this paper}

Please note that where the full-text provided on Manchester Research Explorer is the Author Accepted Manuscript or Proof version this may differ from the final Published version. If citing, it is advised that you check and use the publisher's definitive version.

\section{General rights}

Copyright and moral rights for the publications made accessible in the Research Explorer are retained by the authors and/or other copyright owners and it is a condition of accessing publications that users recognise and abide by the legal requirements associated with these rights.

\section{Takedown policy}

If you believe that this document breaches copyright please refer to the University of Manchester's Takedown Procedures [http://man.ac.uk/04Y6Bo] or contact uml.scholarlycommunications@manchester.ac.uk providing relevant details, so we can investigate your claim.

\section{OPEN ACCESS}




\section{Do Perceptual Instructions Lead to Enhanced Performance Relative to Behavioral Instructions?}

\begin{tabular}{|r|l|}
\hline Journal: & Journal of Motor Behavior \\
\hline Manuscript ID & $35-17-032-R A . R 1$ \\
\hline Manuscript Type: & Research article \\
\hline Date Submitted by the Author: & n/a \\
\hline Complete List of Authors: & $\begin{array}{l}\text { Brown-Ojeda, Carla; University of Manchester, School of Health Sciences } \\
\text { Mansell, Warren; University of Manchester, }\end{array}$ \\
\hline Keywords: & motor performance, instructions, perception, closed-loop \\
\hline \multicolumn{2}{|l}{} \\
\hline
\end{tabular}


Do Perceptual Instructions Lead to Enhanced Performance

Relative to Behavioral Instructions?

Carla Brown-Ojeda \& Warren Mansell

School of Health Sciences, University of Manchester, Manchester, UK.

Running Head: INSTRUCTION AND PERFORMANCE

Correspondence concerning this article should be addressed to:

Dr Warren Mansell, CeNTrUM (Centre for New Treatments and Understanding in Mental Health), Division of Psychology and Mental Health, School of Health Sciences, Faculty of Biology, Medicine and Health, University of Manchester, Manchester Academic Health Science Centre, 2nd Floor Zochonis Building, Brunswick Street, Manchester, M13 9PL, UK Tel: 01612758589

$\square$ Email address: warren.mansell@manchester.ac.uk 


\begin{abstract}
We commonly instruct people in terms of physical actions to carry out in order to perform any task. However, according to Perceptual Control Theory (PCT; Powers, 1973), human action serves to control perception. Using a drawing task, it was hypothesized that instructions describing the perceivable effects of the movement would result in greater accuracy of image drawing than those describing the movement. Participants were asked to draw images using different instructions; describing the expected perceptions and describing the movements. As predicted, it was found that describing the perceptual outcome of the task led to significantly more accurate drawings than describing the movements. This highlights the importance of instructions based on desired perceptions when precise execution of a skill is necessary.
\end{abstract}

Keywords: Skill performance, action, motor, perception, control theory. 
Verbal instructions are used in everyday life to guide people in the execution of many skills. It is might be expected that it is more helpful to give instructions that describe the movements to carry out - the instructor telling the listener 'what to do'. However is this the case? A vast scientific literature exists around skill/task performance and the enhancing effects of different instructions. In particular it has been applied to enhancing sport performance (e.g. Williams \& Hodges, 2005) and rehabilitation techniques used in occupational therapy (Poole, 1991). In terms of basic science, the field of motor behavior has addressed different variables that could contribute to skill performance. In the present study we examine this research, specifically in relation to several theories: knowledge of results (KR; Schmidt, 1982), attentional focus (Wulf, 2007) and perception/action constraints (Hommel, 2009). Secondly, we argue PCT-based task analysis techniques such as PERCOLATe (Marken, 1999) are better than not PCT-based task analysis methods such as HTA (Annett \& Duncan, 1967). Finally, we propose by using PCT methods, we are able to understand the types of instruction that can facilitate performance of a skill. More specifically, it will be argued that instructions describing the desired perceptions upon performance of a skill, rather than the observable actions, will enhance skill performance.

One large body of research has examined how feedback can improve acquisition and retention of skills in certain tasks. This feedback (KR) is often given in a verbal form by the experimenter and describes the success of the motor response in relation to the environmental goal (rather than the movement goal) (Schmidt, 1982). Studies in this area have come to a general consensus that KR improves performance in acquisition phases but is detrimental to performance on future retention tests without KR (see Salmoni, Schmidt \& Walter, 1984 for a review). This has been explained using the guidance hypothesis, which claims that normal learning is inhibited as people rely too heavily on KR (Salmoni et al., 1984). However, KR research seems to focus on learning in the context of feedback given after a performance and 
how participants can change their behavior on future performance using this feedback. For this reason, it does not seem directly relevant towards understanding the type of instructions that facilitate skill performance (i.e. in advance of, or during, the performance of a skill).

A different area of research has focussed on the effects of different instructions prior to task performance (Wulf, 2007). In particular, it has looked at how skill performance (especially in the domain of sport) can be facilitated by adjusting the focus of attention of the performer through instruction provided by a coach. In the first study of this kind, Wulf, Höß, and Prinz (1998) used a ski simulator task to demonstrate that performance improved when participants were told to focus on the pressure exerted onto the wheels (an external focus of attention), as opposed to the pressure exerted by their feet (an internal focus). In other words, by instructing participants to focus on aspects of the environment whilst performing a task led to improved performance on that task. Since then, many different kinds of studies (speed on a pedalo, Totsika \& Wulf, 2003; jump and reach tasks, Wulf, Zachry, Granados \& Dufek, 2007) have demonstrated the beneficial effects on skill performance of directing attention towards external aspects of the environment as opposed to internal body movements.

In order to explain the above findings, Wulf and colleagues proposed the constrained action hypothesis (Wulf, McNevin \& Shea, 2001). According to this, focussing on one's own body movement (internally) interferes with the automatic processes of the motor system. Conversely, focussing on aspects of the environment (externally) encourages fast, unconscious and automatic motor movement. Different lines of indirect evidence that support this theory have been proposed. For example, Wulf et al. (2001) found that when participants were performing two tasks simultaneously (a balancing task and probe reaction task), reaction time was greater on the probe task when participants were told to focus internally (as opposed to externally). Wulf et al. (2001) suggest that this occurs because of the greater attentional demands required to move when focussing on bodily movements. However, the 
authors do not make it clear exactly what these automatic processes are and therefore how exactly different focuses of attention moderates them. It is possible that differences in skill performance could be due to particular aspects of internal or external instructions, such as whether they represent the perceptual results of an action (Shea et al., 2015).

Research into complex motor skills has provided some clearer insight into how perceptions could facilitate motor skill performance, in particular how perceptions/actions inhibit or facilitate performance (Shea et al., 2015). In general, people are better at performing in-phase coordination patterns as homologous muscles are activated during the coordination pattern (Kelso \& De Guzman, 1988). The anti-phase coordination pattern is more complex as non-homologous muscles are activated. When perceptual symmetry is created using a visual display of the motor movements required, performance of the antiphase pattern is just as good (Meschner \& Knoblich, 2014). This suggests that despite difficult motor constraints, increasing perceptual feedback can facilitate the performance. This is further supported by the fact that when people are unable to perceive a coordination pattern that is presented visually, they are unable to perform the pattern effectively (Wilson, Collins \& Bingham, 2005). Additionally, and in concordance with the studies reviewed by Wulf (2007), visually monitoring the limbs has been demonstrated to inhibit bimanual coordination (Shea et al., 2015). In other words, perceptions facilitate skill performance, rather than actions. Conversely, focusing attention on actions appears to inhibit skill performance.

Yet there is a body of evidence that appears to contradict the conclusions regarding a separation of perception and action. One theory, introduced by Hommel, Musseler, Aschersleben and Prinz (2001), known as the theory of event coding, proposes that actions and perceptual events that follow an action are commonly coded. According to Hommel (1996), perceiving the effects of an action creates cognitive structures that mediate both 
action and perception. For example, participants responding to visual stimuli with binary left or right hand responses, respond faster when there is a congruent match between the spatial location of the stimulus and response (Simon \& Ruddell, 1967; Craft \& Simon, 1970). This provides evidence for common coding between the perceived effects of action and the action itself as congruency between the two leads to beneficial effects and incongruency leads to processing delays (Hommel, 1996).

Whilst taking the above literature into account, it is unclear whether instructions that describe actions or perceptual consequences of actions are more effective. Task analysis methods based on feedback models (e.g. Hierarchical Task Analysis (HTA; Annett \& Duncan, 1967) break down tasks into a hierarchy of subordinate task steps. An operator must use feedback signals from their own infrastructure or the environment to evaluate and control their behaviour. This is based on the Test-Operate-Test-Exit model (TOTE; Miller, Galanter \& Pribram, 1960), where every task step is a TOTE unit. However, a disparity in views of HTA's effectiveness exists. The main issue it has relates the to the extent to which the feedback elements of the task are actually recognized and controlled as intended by the operator. For this reason, alternative approaches to task analysis incorporating Perceptual Control Theory (PCT; Powers, 1973) have been made. PCT proposes that any action can be decomposed into its perceptual components. According to PCT, movement occurs to control the perceptual variables that are necessary to complete any skill successfully. Action and perception are not commonly coded. Rather, actions serve to control our perceptions, which occurs in a closed loop. The components and organization of this loop have been specified and modeled (e.g. Powers, 1973, 1978). In brief, any skill can be deconstructed into its perceptual goals, at multiple levels. This goes one step further than any non-PCT based task analysis as PCT describes behaviour in a way of controlling perception, rather than controlling a stimulus itself. For example, a PCT- based task analysis method, named 
PERCOLATe is used to decompose a task into its corresponding perceptual control characteristics (Marken, 1999). These control characteristics are perceptual representations of physical variables that need to be maintained in a preferred state in order to complete the task. To do this an operator must compare the sensory input (what they are perceiving) with the reference input (what they want to perceive i.e. the end goal of the task) and calculate an 'error'. Action must occur in order to correct the 'error'. In other words, perception is both a product and driver of behavior. Further PCT-based task analysis methods have also demonstrated this such as PCHAT (Phipps \& Harris, 2014). This seems to suggest therefore that the most accurate performance of a skill will be when the reference points for perceptual variables are specified. In contrast, specifying the actions from an observer's viewpoint will result in less accurate performance because these typically do not map onto the perceptual variables required to perform the skill.

Previous research has not attempted to completely separate the perceptual representation of a skill from the observable behavior and compare and measure their effectiveness on task performance. The present study aimed to test the hypothesis that perceptual instructions (describing what the participant can expect to perceive) leads to greater accuracy in a drawing task than action instructions (describing what movements to carry out). In the control conditions the participant were shown a picture of what they should draw and asked to draw it from vision or memory. This was used to compare the accuracy of drawing when the perceptual variables are made as explicit as possible. 
INSTRUCTION AND PERFORMANCE

\section{Method}

\section{Participants}

The study received ethical approval through the university. A sample of 30 female and two male Psychology students were recruited through an online participation scheme. Students participated in exchange for course credits. The mean age of participants was 19.84 $($ range $=18-36)(S D=3.16)$. Half of the participants were White British $(53.1 \%)$ and the other half were made up of; $18.8 \%$ White other, 6.3\% Asian Indian, 3.1\% Asian Pakistani, 12.5\% Asian other, 3.1\% Arabic and 3.1\% preferred not to say. Students with previous Chinese symbol expertise were requested not to volunteer as they would be familiar with the Chinese symbols used in this study. For the purpose of the study, the Chinese symbols were intended to be novel so that a prior perceptual representation of the image did not exist. Therefore they would be at an advantage for completing the task. To ensure consistency, only right-handed students were recruited.

\section{Design}

A 4 x 3 within-subjects factorial design was used. The first independent variable was Instruction. This described how the participant should draw the image and there were four levels: Direct copy (C), copy from memory (M), draw using action instructions (A) and draw using perception instructions $(\mathrm{P})$. The second independent variable was Complexity, which had three levels: Simple, Familiar and Complex. Four categories of images, consisting of three images each (one for each complexity) were used. In order to counterbalance, these were combined in four different orders with four different orders of instructions (CMPA, MPAC, PACM and ACMP). This resulted in 16 conditions. The 16 conditions were labeled 1-16, selected at random (using excel) and then assigned to each participant under the constraint that each condition was assigned two participants. The dependent variable was accuracy of drawings. 
INSTRUCTION AND PERFORMANCE

Materials

Images

A set of 12 images printed on white $6 \mathrm{~cm} \times 6 \mathrm{~cm}$ cards was used. For each Complexity level (Simple, Familiar, Complex) four images were chosen. Common shapes e.g. a square, were chosen for Simple Complexity. These represented images that were well known and commonly encountered. Familiar 2D images of objects were used for Familiar Complexity e.g. a plane. These images were chosen to be harder to draw, but still most likely encountered by the participants before. Finally, novel images of Chinese symbols were chosen to represent Complex Complexity. The purpose of using Chinese symbols was that participants would not have prior visual representations of these images in memory. The images chosen were similar in size and difficulty within each Complexity level.

\section{Action and perception instructions}

Each image had a corresponding action and perception instruction. The action instructions described the movements to carry out in order to draw the image, i.e., how the participant should move his or her hand. For example, the action instruction used for drawing a triangle was: "Place your pen in the middle at the top of the page and draw for 4 seconds towards the bottom left corner of the page. Keeping your pen on the page, draw across to the right side of the page for 3 seconds". The instructions were designed and modified using piloting. The purpose of the action instructions was to eliminate the use of perceptual references when drawing each shape. However, during piloting, many of the instructions were impossible to follow accurately without these. For this reason reference points such as "the middle of the page" were deemed necessary for the instruction to at least be possible.

The perception instructions described what the participant could expect to perceive. For example the equivalent perception instruction for the triangle was: "Draw an isosceles triangle with sides $4 \mathrm{~cm}$ by $3 \mathrm{~cm}$ by $4 \mathrm{~cm}$ ". These were designed to describe what the 
participant could expect to see on the page. All instructions were reviewed in piloting; any information regarding movement was removed.

\section{Word count and readability}

Action instructions had a median word count of 116.50 (Interquartile range (IQR) $=$ 93.50). Their median Flesch readability score was $85.05(\mathrm{IQR}=6.80)$. Word count and readability medians for perception instructions were $41.00(\mathrm{IQR}=74.25)$ and $90.05(\mathrm{IQR}=$ 10.08) respectively. Both median readability scores are considered easy. Wilcoxon signed ranks was used to test whether these differed between instructions. Word count was significantly different for action and perception instructions $(\mathrm{T}=3.00, \mathrm{p}=.005)$. However, readability was not significantly different $(\mathrm{T}=25.00, p=.272)$.

\section{Rating scales}

Three rating scales were printed on a single sheet of paper. These were: 1) "How difficult were these instructions to follow?" (Difficulty) 2) “To what extent do these instructions describe the movements_you should carry out?” ('Movement') 3) “To what extent do these instructions describe what you should expect to see on the page?” ('See'). The maximum score on the scale for difficulty was ten (extremely difficult) and the minimum was zero (extremely easy). The maximum score for 'movement' and 'see' was also ten (completely) and the minimum was zero (not at all).

\section{Accuracy scale}

Three scales for each individual component of image accuracy were used to rate the accuracy of each drawing. See Table 1. Preliminary trials suggested a four-point scale was most appropriate as it provided higher repeatability than higher resolution scales. It was also found that summed scores of size-scale point by point distances between the original and copy would not be practically possible due to the possibility that some drawings would be completely different to the original, making it impossible to find comparison points. All three 
INSTRUCTION AND PERFORMANCE

components were combined to give a total accuracy score out of 12. All components were independent from each other. For example, a picture of a square could be given a score of 3 for features (all features there, i.e., all four lines there) but a score of 0 for resemblance (impossible to see resemblance, i.e., if the lines of the square were not connected).

---- PLEASE INSERT TABLE 1 AROUND HERE ---

\section{Procedure}

Participants were told that the purpose of the study was to test how well they could draw different images using different types of instructions. Participants were told to draw each image on a separate square of paper using the black marker pen. They were also instructed to mark errors with a red line. For the Copy instruction, the participant was asked to look at the image for 30 seconds (timed on a stopwatch) before beginning to draw it. They were told they could look back at the image after the 30 seconds if they wanted. This was repeated three times for an image of each complexity. For the Memory instruction, they were told to memorize an image for 30 seconds. After the 30 seconds, the image was taken away. Participants were instructed to draw the image from memory. Again this was repeated for an image of each complexity. For both action and perception instructions, participants were told to not begin drawing until they had read the instruction for at least 30 seconds. They were told they could look back at the instruction throughout drawing. This was repeated for each image of different complexity. Each participant drew a total of 12 images. The order of instructions differed depending on the condition the participant had been assigned to. Next, each action and perception instruction was handed back to the participant to rate the difficulty, 'movement' and 'see' scale. These were given one at a time and the participant was told to re-read the instruction for at least 30 seconds before rating it. This was done to ensure participants actually read the instruction and therefore thought more carefully about each scale. At the end of the study participants were asked how they had found the study. 
INSTRUCTION AND PERFORMANCE

\section{Rating accuracy of drawings}

Two raters were given a training session, which consisted of practicing the rating of 12 shapes reproduced in pilot studies, using the accuracy scale. Their scores were reviewed and whenever a discrepancy occurred they were asked to explain why they had given a certain score and subsequently corrected. Once satisfied by the rater's accuracy they were then given the full set of images to code over two ( 1 and a half hour) periods (see Results).

\section{Results \\ Manipulation check \\ A manipulation check was done to check the validity of each action and perception instruction. Participant's ratings were compared for: 1) ratings of 'movement' for action and perception instructions and 2) ratings of 'see' for action and perception instructions.}

As data were not normal a Wilcoxon signed rank test was used to compare instructions. For simple shapes, 'movement' ratings were significantly different for action $($ median $=8.00, \mathrm{IQR}=2.50)$ and Perception $($ median $=3.00, \mathrm{IQR}=6.75)$ instructions $(\mathrm{T}=$ 33.00, $p<.001)$. There was no significant difference between 'movement' ratings for familiar action $($ median $=7.50, \mathrm{IQR}=5.00)$ and familiar perception $($ median $=5.50, \mathrm{IQR}=5.00)$ instructions $(\mathrm{T}=148.50, \mathrm{p}=.134)$ and between complex action $($ median $=8.00, \mathrm{IQR}=3.00)$ and complex perception (median $=7.00, \mathrm{IQR}=3.00)$ instructions $(\mathrm{T}=136, p=.125)$. 'See' ratings were significantly different for action and perception instructions regardless of complexity; simple action (median $=2.00, \mathrm{IQR}=6.75)$ vs. simple perception $($ median $=$ $10.00, \mathrm{IQR}=1.75),(\mathrm{T}=2.50, p<.001)$, familiar action $($ median $=1.00, \mathrm{IQR}=2.00) \mathrm{vs}$. familiar perception $($ median $=9.00, \mathrm{IQR}=2.00),(\mathrm{T}=.00, p<.001)$ and complex action $($ median $=1.00, \mathrm{IQR}=2.00)$ vs. complex perception $($ median $=6.00, \mathrm{IQR}=6.00),(\mathrm{T}=$ $19.00, p<.001)$. Sample size was $n=32$ for all tests.

\section{Inter-rater reliability}


INSTRUCTION AND PERFORMANCE

A total of 12 intraclass correlation coefficients were calculated to compare rater agreements for each complexity and instruction combination. The coefficients ranged from $.505-.875$, with $p \leq .001$ in every case.

\section{Accuracy}

As the range for the individual components (size/features/resemblance) was too small for any statistical test, accuracy was taken as the sum of the scores from all three. As data for accuracy for each combination of instruction and complexity were not normal, complexity was pooled for each instruction. This provided normally distributed variables, allowing a one-way Repeated Measures ANOVA to be used to test the differences in accuracy under each Instruction. The main hypothesis for this study was that accuracy of drawing would be greater for perception rather than action instructions. The analysis demonstrated a significant difference between the condition means $F(1.79,55)=162.70, p<.001$ (Greenhouse-Geisser test as the assumption of sphericity was violated). This represented an effect size ( $\square \square \square \square^{2}$ ) of .84 , i.e., $84 \%$ of the variance in accuracy was accounted for by Instruction. Pairwise posthoc analyses using Bonferroni correction confirmed the hypothesis: accuracy ratings were significantly greater for perception instructions over action instructions $(p<.001)$ and demonstrated a large effect size $(d=2.66)$. See Figure 1 for a graph showing all condition differences. See Figure 2 for a table showing actual participant drawings.

To further examine the robustness of these results, Wilcoxon signed ranks was used to test the differences between action and perception instructions under the different complexities of the shapes, without assuming normality. The accuracy ratings were significantly different for action and perception instructions regardless of complexity: simple $(\mathrm{T}=41.50, p<.001)$, familiar $(\mathrm{T}=1.00, p<.001)$ and complex $(\mathrm{T}=39.00, p<.001)$.

\section{Difficulty}


Difficulty of the instructions was tested as a possible confound variable using the ratings given by participants. As the data were not normal, the ratings were pooled across complexity. This achieved normalization and so the data were analyzed using a paired t-test. This revealed that the mean of the differences between difficulty ratings for action $(M=$ 16.53, $S D=5.93)$ and perception instructions $(M=8.41, S D=4.2)$ differed significantly; $t(31)=7.02 ; p<.001$. The mean difference, 8.13 (95\% CI [5.76-10.49]) demonstrated a large effect size, $d=1.61$.

Again, to further investigate this difference without assuming normality, Wilcoxon signed ranks was used to test these differences under the different complexities of the shapes, for the non-normal data. The difficulty ratings for action instructions (simple shape median = $3.50, \mathrm{IQR}=3.00$, familiar shape median $=7.00, \mathrm{IQR}=2.75$ and complex shape median $=$ 7.00, IQR $=3.75$ ) and perception instructions ( familiar shape median $=3.00, \mathrm{IQR}=2.75$ and complex shape median $=3.00, \mathrm{IQR}=3.00$ ) were significantly different regardless of complexity: simple $(\mathrm{T}=32.00, p<.001)$ familiar $(\mathrm{T}$ $=29.00, p=.001)$ and complex $(\mathrm{T}=83.50, p<.001, d=1.13)$. 


\section{Correlation between difficulty, accuracy, 'movement' and 'see'.}

Given that difficulty ratings differed between the type of instruction, spearman's rank correlation coefficients were obtained in order to investigate whether difficulty correlated with accuracy within these conditions. Contrary to the view of difficulty as a potential confounder, none of the rank correlations between difficulty and accuracy were significant, regardless of instruction or complexity.

In addition, Spearman's rank correlations were also obtained for 'movement'/'see' ratings and difficulty to see whether there was an association between either of the ratings. There was a significant negative correlation between 'movement' ratings and difficulty for simple complexity, action instructions $($ rho $=-.45)$. There were also significant negative correlations between difficulty and 'see' ratings for all perception instructions regardless of complexity (rho $=-.45$ to -.43 ). Additionally, although 'see' ratings correlated significantly with difficulty, for simple shapes, 'movement' ratings correlated with difficulty. 
Discussion

The present study examined the effect of changing the type of instruction on accuracy of drawing different images. Specifically, it investigated whether giving participants perceptual instructions allowed them to draw more accurately than when they were given action instructions. The results provided support for the hypothesis that perceptual instructions led to greater accuracy of drawing compared with 'movement' instructions. Provision of perceptual references increased the accuracy of the reproduced images compared with simply describing the movements required. This finding was consistent, even when an unfamiliar image was used (complex image), and therefore likely no prior perceptual representation existed. Interestingly, the mean accuracy score for perceptual instructions was high, approaching that of drawings that were directly copied or copied from memory.

Overall, the findings from this study are in line with our prediction: describing the suitable perceptions that need to be controlled allows the listener to perform the task more accurately. Furthermore, as expected from a PCT based task analysis (e.g. PERCOLATe), the operator of any task would be regarded as a perceptual control system, whose goal is to control the perceptual components of the task (Marken, 1999). In the present study, participants were using their hand movements (actions) in order to reach the desired goal of the task (the image to be drawn). Instructing participants on what to perceive allowed participants to monitor their sensory input and calculate an error in relation to the reference state (i.e. the image to be drawn). Conversely, instructing participants on appropriate actions appear to have made participants perform poorly as these instructions did not map on to the perceptual variables required to perform the skill. This demonstrates the effectiveness of a PCT-based task analysis, where when the task is decomposed into perceptual variables is much more useful than simply providing action related task steps. 
Although the results from the present study might have been predicted by the work of previous researchers (e.g. Wulf et al., 2001; Hommel et al., 2001), the study goes one step further than the aforementioned studies. The present study broke down the task into various task components, either perceptual or motor ones, demonstrating the importance of perceptual task components in order to enhance performance. The constrained action hypothesis, although theoretically similar, only goes as far as to say that internal focus of attention of the person inhibits the 'automatic' processes of the nervous system. However, from our study we would argue that task performance goes further than the constrained action hypothesis. In other words the perceptual components of any task will vary and consist of different perceptions. In relation to the present study, describing what the participant could expect to see allowed them to use their movements to control this perception, resulting in a more accurate drawing, rather than the spatial location of the attentional focus.

It is important to note that these conclusions do not represent a criticism of the robustness of the findings by Wulf and colleagues (e.g. Wulf et al., 2001; Wulf \& Lewthwaite, 2016). Nor indeed do they challenge the proposal that externally focused attention is typically advantageous to motor performance because it may establish efficient connections across brain networks and prevent 'backsliding' towards aspects of the self that are not relevant to the task (Wulf \& Lewthwaite, 2016). Rather, we propose that it is not the internal/external aspect of such instructions that brings about a difference in task performance. Some tasks, such as shooting basketball hoops, may consist of perceptual variables that are available in the environment and therefore would benefit from an external focus, e.g., the rim of the basketball hoop (as found by Zachary et al., 2005). However, other tasks may consist of internal perceptual components, for example performing a dance move, and therefore benefit from instructions describing internal perceptions, such as pressure on parts of the feet. In other words, it is not the internal or external aspect of an instruction that 
enhances performance but rather the extent to which the perceptual components (that make up a task) are made clear.

This brings us to PCT, which suggests action occurs to control the perceptions that are required to complete a specific task. By making these perceptions as clear as possible in a task, a person can complete the task more efficiently (as demonstrated in the present study). This is consistent with findings by Meschner and Knoblich (2004) who found complex motor coordination patterns were facilitated by perceptions. However, it seems likely that rather than action and perception being commonly coded (as suggested by Hommel, 1997 and adopted by Meschner \& Knoblich, 2014), our actions control our perceptions in a closed loop and therefore we are better at performing a task when the perceptual components that make up that task are specified.

In the present study, different aspects of the instructions (difficulty, word count and Flesch readability) were measured to test possible confounds that could explain the pattern of results obtained. It was found that action instructions (those describing the movements) were rated significantly more difficult than perception instructions (those describing what to perceive). This was examined further by correlating difficulty with each of our manipulation checks ('see' and 'movement'). It was found that difficulty was negatively correlated with 'see' ratings. In other words, as participants were less able to perceive what they were supposed to draw, the more difficult they found the instructions to follow. As expected, this would suggest that the differences in difficulty were caused by the differences in being able to perceive what to draw. Secondly, upon examination of the correlations between accuracy and difficulty, none were found to be significant, suggesting that difficulty per se had little or no impact on the results. Additionally, Flesch readability percentages indicated that all instructions were either very easy, easy or fairly easy to read and did not differ significantly between instruction type. Interestingly, word count was significantly smaller for perceptual 
instructions. A possible interpretation of this could be that even with less information, participants performed better on the task. However, in future, it would be important to try and control the amount of information given for each instruction to try and equate it between instruction types.

From the manipulation check it was found that participant's ratings for familiar and complex instructions did not differ significantly for 'movement' ratings between action and perception instructions. This is interesting as during the design and evaluation of the perceptual instructions, it was noticed that some of the instructions for the complex images were contaminated with 'movement' information. It was thought that most of the information was removed but perhaps some instructions still appeared to describe the movements. A possible interpretation of this could be that as a task becomes more difficult and no prior perceptual representation exists, internal perceptions relating to the movements required by the task may be more useful. Or, a combination of different perceptions may be more useful in novice tasks, e.g., movement perceptions as well as visual perceptions. It would be interesting for future research to investigate this further. Secondly, the action instructions were not purely action. For example, the majority described reference points on the page e.g. "the middle" or "the top left corner". Ideally, these would not have included any perceptual references, however during piloting of the study it became clear that it was impossible to communicate movement without at least stating where on the page to begin the movement. Further to this point, it could also be argued that even time and velocity are perceptual references as they are something that can be perceived. If one were to analyze the action instructions in depth, then a strong case could be made that the action instructions were “infected" with perceptual references, however this only supports our argument further as common theories propose we control action outputs (e.g. Miall \& Wolpert, 1996) but how can this be the case if all actions require the specification of their perceptual results to be 
carried out effectively? This only seems to further support our point of the importance of perceptions in performing a task accurately.

Some limitations should be acknowledged. Firstly, it is important to note that the perceptual instructions for complex images were often described in a way that broke down the image into "familiar" perceptions, e.g., "Draw a $6 \mathrm{~cm}$ long vertical line down the middle of the page, around the middle of this line draw a square with $2 \mathrm{~cm}$ long sides" (complex image Y). It could be argued therefore that the complex images were not "unfamiliar" to the participant as they were intended. However, we would argue that the participant had no prior full visual representation of the image in memory. Secondly, the present study does not imply that the only thing necessary for drawing e.g. a square, is to know what a square looks like. Certain skills need to be developed in order to be able to draw a square. According to Van De Rijt-Plooij and Plooji (1992), humans transition through different regression periods in childhood during which control over increasingly complex perceptual variables emerge within a hierarchical organisation. It is not until a child has developed these systems that she is able to acquire certain complex skills such as drawing. However, this study did not aim to test the developmental foundation of skills. Rather it tested how one can communicate instructions describing how to draw an image in adults that have already fully developed systems for doing so.

Following on from the above point, one might postulate that the advantageous effects of external focus of attention (Wulf \& Lewthwaite, 2016) might result from the perceptual specification of the controlled variables for the skill being at the top of a hierarchical organisation. At this point, the brain can draw upon multiple brain networks to achieve a desired end state, rather than the attention being focused on the perceptual specifications lower down in the hierarchy which might overemphasize specific methods of how to perform the task, thereby limiting its flexibility to the changing circumstances during performance. 
INSTRUCTION AND PERFORMANCE

The design required to examine this hypothesis would need to first decompose a novel motor task into a hierarchy of its potential perceptual variables and their reference states - e.g. a block design test in which different patterned blocks must be arranged in the right orientation and position relative to one another to make the specified overall pattern. Once this hierarchy is established, participant performance would be compared under conditions in which the focus of attention is manipulated towards perceptual variables at different levels in the hierarchy, whilst the participant is subjected to known disturbances to performance - e.g. a rotating table; changes in lighting conditions. It would be predicted that attention towards the variables at the top of the hierarchy would be more advantageous than attention towards those at the bottom, regardless of whether the perceptual variables are considered to be external or internal.

The present research has many interesting implications, in particular in relation to occupational therapy. Many studies have attempted to apply the different findings from motor behavior research to enhance rehabilitation techniques after strokes (Van Vliet \& Wulf, 2006) and improve balance in patients with Parkinson's disease (Landers, Wulf, Wallmann \& Guadagnoli, 2005; Wulf, Mercer, McNevin \& Guadagnoli, 2004). Although some studies have found that different focuses of attention can enhance balance in patients with Parkinson's disease (e.g. Landers et al, 2005), many have been inconclusive (e.g. Walker, Brouwer \& Culham, 2000; Kilduski \& Rice, 2003). During rehabilitation, everyday skills such as walking, standing and grasping an object need to be taught. This study suggests that helping patients to learn the perceptual variables that need to be controlled when performing these skills could enhance rehabilitation regardless of whether they are internal or external. For this reason, it would be useful for future research to decompose these skills into their perceptual components in order to provide the most beneficial instructions, in terms of the perceptions that should be controlled during the performance of the skill. 
This study appears to be the first of its kind, testing the effect of describing how to perform a skill in terms of the perceptual variables compared to the observable actions. Instructing participants on what they can expect to perceive has significantly greater advantage for reproducing images than instructing participants on the movements they should make. The results are in line with a PCT-based task-analysis approach that specifies the importance of perceptual feedback in the successful performance of a task. It is therefore concluded that any task requiring a precise execution should be broken down into it perceptual components. The person performing the task should then be instructed according to these components (when using verbal instruction). Future research should aim to break down different tasks into their perceptual variables in order to use the most appropriate variables in instruction. 
References:

Annett, J., \& Duncan, K. D. (1967). Task analysis and training design. Occupational Psychology. 41, 211-221

Hommel, B. (1996). Toward an action-concept model of stimulus-response compatibility. In B. Hommel \& W. Prinz (Eds.), Theoretical issue of stimulus-response compatibility (pp. 281-320). Amsterdam: North-Holland.

Hommel, B. (2009). Action control according to TEC (theory of event coding). Psychological Research PRPF, 73(4), 512-526.

Hommel, B., Müsseler, J., Aschersleben, G., \& Prinz, W. (2001). Codes and their vicissitudes. Behavioral and brain sciences, 24(05), 910-926.

Kelso, J. A. S. (1988). Order in time: How the cooperation between the hands informs the design of the brain. In H. Haken (Ed.), Neural and synergetic computers (pp. 180196). Berlin: Springer.

Kilduski, N. C., \& Rice, M. S. (2003). Qualitative and quantitative knowledge of results: effects on motor learning. American Journal of Occupational Therapy, 57(3), 329336. 
Landers, M., Wulf, G., Wallmann, H., \& Guadagnoli, M. (2005). An external focus of attention attenuates balance impairment in patients with Parkinson's disease who have a fall history. Physiotherapy, 91(3), 152-158.

Lashley, K. S. (1951). The problem of serial order in behavior. In L. A. Jeffress (Ed.), Cerebral mechanisms in behavior (pp. 112-136). New York: Wiley.

Marken, R. S. (1986). Perceptual organization of behavior: a hierarchical control model of coordinated action. Journal of Experimental Psychology: Human Perception and Performance, 12(3), 267-276.

Marken, R. S. (1999). PERCOLATe: perceptual control analysis of tasks. International Journal of Human-Computer Studies, 50(6), 481-487.

Marken, R. S., Mansell, W., \& Khatib, Z. (2013). Motor control as the control of perception. Perceptual \& Motor Skills, 117(1278), 10-2466.

Mechsner, F., \& Knoblich, G. (2004). Do muscles matter for coordinated action. Journal of Experimental Psychology: Human Perception and Performance, 30(3), 490-503. 
INSTRUCTION AND PERFORMANCE

Mechsner, F., Kerzel, D., Knoblich, G., \& Prinz, W. (2001). Perceptual basis of bimanual coordination. Nature, 414(6859), 69-73

Miall, R. C., \& Wolpert, D. M. (1996). Forward models for physiological motor control. Neural networks, 9(8), 1265-1279.

Miller, G. A., E. Galanter, and K. H. Pribram. 1960. Plans and the Structure of Behavior. London: Holt, Rinehart and Winston.

Phipps, D. L., \& Harris, D. (2014). Perceptual control and feedback control in the analysis of complex tasks. Theoretical Issues in Ergonomics Science, 15(5), 505-516.

Poole, J. L. (1991). Application of motor learning principles in occupational therapy. American Journal of Occupational Therapy, 45(6), 531-537.

Powers, W. T. (1973). Behavior: The control of perception. Chicago: Aldine.

Powers, W. T. (1978). Quantitative analysis of purposive systems: Some spadework at the foundations of scientific psychology. Psychological Review, 85(5), 417-435.

Powers, W. T., Clark, R. K., \& Mc Farland, R. L. (1960). A general feedback theory of human behavior: Part I. Perceptual and Motor Skills, 11(1), 71-88. 
Powers, W. T., Clark, R. K., \& Mc Farland, R. L. (1960). A general feedback theory of human behavior: Part II. Perceptual and Motor Skills, 11(3), 309-323.

Salmoni, A. W., Schmidt, R. A., \& Walter, C. B. (1984). Knowledge of results and motor learning: a review and critical reappraisal. Psychological Bulletin, 95(3), 355-386.

Schmidt, R. A. (1982). Motor control and learning: A behavioral emphasis. Champaign, IL: Human Kinetics Press.

Shea, C. H., Buchanan, J. J., \& Kennedy, D. M. (2015). Perception and action influences on discrete and reciprocal bimanual coordination. Psychonomic Bulletin \& Review, 23(2), 361-386.

Simon, J. R., \& Craft, J. L. (1970). Effects of an irrelevant auditory stimulus on visual choice reaction time. Journal of Experimental Psychology, 86(2), 272-274.

Simon, J. R., \& Rudell, A. P. (1967). Auditory SR compatibility: the effect of an irrelevant cue on information processing. Journal of Applied Psychology, 51(3), 300-304.

Totsika, V., \& Wulf, G. (2003). The influence of external and internal foci of attention on transfer to novel situations and skills. Research Quarterly for Exercise and Sport, 74(2), 220-232. 
INSTRUCTION AND PERFORMANCE

Van De Rijt-Plooij, H. H., \& Plooij, F. X. (1992). Infantile regressions: Disorganization and the onset of transition periods. Journal of Reproductive and Infant Psychology, 10(3), 129-149.

Van Vliet, P. M., \& Wulf, G. (2006). Extrinsic feedback for motor learning after stroke: what is the evidence?. Disability and Rehabilitation, 28(13-14), 831-840.

Walker, C., Brouwer, B. J., \& Culham, E. G. (2000). Use of visual feedback in retraining balance following acute stroke. Physical Therapy, 80(9), 886-895.

Wilson, A. D., Collins, D. R., \& Bingham, G. P. (2005). Perceptual coupling in rhythmic movement coordination: stable perception leads to stable action. Experimental Brain Research, 164(4), 517-528.

Wulf, G. (2007). Attentional focus and motor learning: A review of 10 years of research. Bewegung und Training, 1(2-3), 1-11. Retrieved from http://www.ejournal-but.de

Wulf, G., Höß, M., \& Prinz, W. (1998). Instructions for motor learning: Differential effects of internal versus external focus of attention. Journal of Motor Behavior, 30(2), 169179. 
Wulf, G., \& Lewthwaite, R. (2016). Optimizing performance through intrinsic motivation and attention for learning: The OPTIMAL theory of motor learning. Psychonomic bulletin \& Review, 23(5), 1382-1414.

Wulf, G., McNevin, N., \& Shea, C. H. (2001). The automaticity of complex motor skill learning as a function of attentional focus. The Quarterly Journal of Experimental Psychology: Section A, 54(4), 1143-1154.

Wulf, G., Zachry, T., Granados, C., \& Dufek, J. (2007). Increases in jump-and-reach height through an external focus of attention. International Journal of Sports Science and Coaching, 2(3), 275-284.

Williams, A. M., \& Hodges, N. J. (2005). Practice, instruction and skill acquisition in soccer: Challenging tradition. Journal of Sports Sciences, 23(6), 637-650. 
Table 1. A table to show the accuracy scale used to rate accuracy of participants drawings compared to the original image.

\begin{tabular}{|l|l|l|l|}
\hline Score & Size & Features & Resemblance \\
\hline 0 & $\begin{array}{l}<25 \% / 300 \%+\text { of } \\
\text { actual size }\end{array}$ & $\begin{array}{l}\text { All features } \\
\text { missing }\end{array}$ & $\begin{array}{l}\text { Impossible to see } \\
\text { resemblance }\end{array}$ \\
\hline 1 & $\begin{array}{l}200 \% / 50 \% \text { of } \\
\text { actual size }\end{array}$ & $\begin{array}{l}\text { Only a few } \\
\text { features there }\end{array}$ & $\begin{array}{l}\text { Only slight } \\
\text { resemblance to } \\
\text { original }\end{array}$ \\
\hline 2 & $\begin{array}{l}125 / 75 \% \text { of actual } \\
\text { size }\end{array}$ & $\begin{array}{l}\text { Most features } \\
\text { there }\end{array}$ & $\begin{array}{l}\text { Can see } \\
\text { resemblance but } \\
\text { not perfect }\end{array}$ \\
\hline 3 & $100 \%$ actual size & All features there & $\begin{array}{l}\text { Identical to } \\
\text { original }\end{array}$ \\
\hline
\end{tabular}


Figure 1. A graph to show the mean and maximum accuracy score under each instruction type.

Note. Bar heights represent mean accuracy under each condition. Vertical lines represent error bars. Horizontal line represents maximum accuracy score.

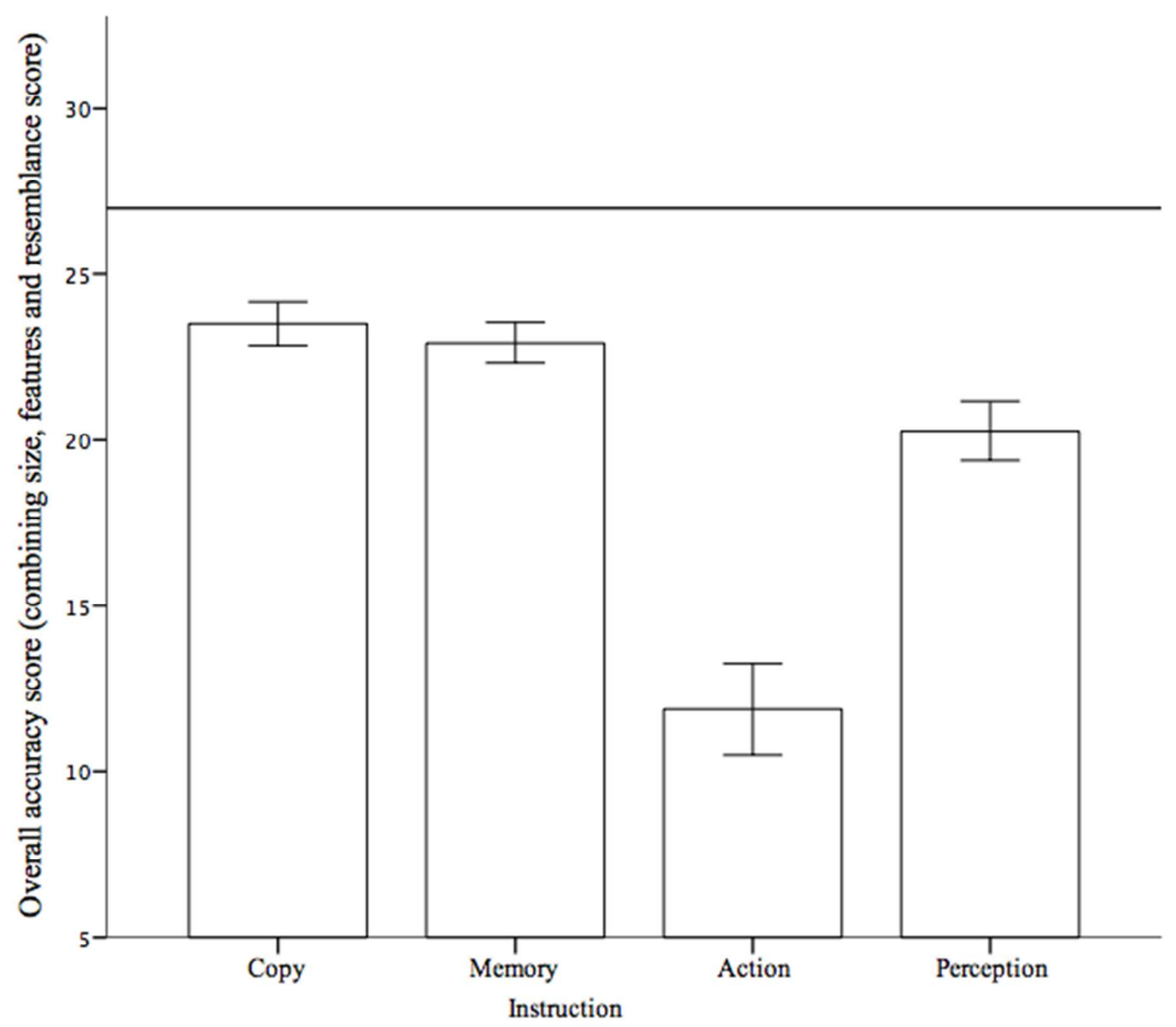


Figure 2. Participant drawings under different instruction types compared to the intended image to be drawn.

\begin{tabular}{|l|l|l|l|l|l|}
\hline $\begin{array}{l}\text { Complexity } \\
\text { of image }\end{array}$ & $\begin{array}{l}\text { Image to be } \\
\text { drawn }\end{array}$ & Direct copy & $\begin{array}{l}\text { Copy from } \\
\text { memory } \\
\text { instructions }\end{array}$ & $\begin{array}{l}\text { Perceptual } \\
\text { instructions }\end{array}$ \\
\hline Simple & &
\end{tabular}


1

2

3

4

5

6

7

8

9

10

11

12

13

14

15

16

17

18

19

20

21

22

23

24

25

26

27

28

29

30

31

32

33

34

35

36

37

38

39

40

41

42

43

44

45

46

47

48

49

50

51

52

53

54

55

56

57

58

59

60 